Check for updates

Cite this: RSC Adv., 2018, 8, 32637

Received 5th June 2018

Accepted 17th August 2018

DOI: $10.1039 / c 8 r a 04777 d$

rsc.li/rsc-advances

\section{Naphthoquinones of the spinochrome class: occurrence, isolation, biosynthesis and biomedical applications}

\begin{abstract}
Yakun Hou, (D) ${ }^{a}$ Elena A. Vasileva, ${ }^{\text {b }}$ Alan Carne, ${ }^{c}$ Michelle McConnell, ${ }^{\text {d }}$ Alaa El-Din A. Bekhit (iD ${ }^{a}$ and Natalia P. Mishchenko*b

Quinones are widespread in nature and have been found in plants, fungi and bacteria, as well as in members of the animal kingdom. More than forty closely related naphthoquinones have been found in echinoderms, mainly in sea urchins but occasionally in brittle stars, sea stars and starfish. This review aims to examine controversial issues on the chemistry, biosynthesis, functions, stability and application aspects of the spinochrome class, a prominent group of secondary metabolites found in sea urchins. The emphasis of this review is on the isolation and structure of these compounds, together with evaluation of their relevant biological activities, source organisms, the location of origin and methods used for isolation and identification. In addition, the studies of their biosynthesis and ecological function, stability and chemical synthesis have been highlighted. This review aims to establish a focus for future spinochrome research and its potential for benefiting human health and well-being.
\end{abstract}

\section{Introduction}

Rational use of biological resources of the aquatic environment is a realistic scientific and practical goal since they represent $70 \%$ of the organisms on earth. Marine hydrobionts, such as sea urchins, and specifically their gonads, are a valuable renewable food resource. At the same time, they can serve as a unique source of various natural compounds, which can be the basis for the creation of various biomaterials, ${ }^{\mathbf{1 , 2}}$ effective medicinal and parapharmaceutical preparations ${ }^{3}$ as well as functional food products. ${ }^{3-5}$ Many species of regular sea urchins are commercially harvested because their gonads are consumed in many countries of Asia, the Mediterranean and North America. After the removal of the gonads, large amounts of sea urchin shells are left as waste. This shell material is rich in bioactive quinonoid pigments, principally spinochromes. ${ }^{4-6}$ Although this class of compounds has been known for over 100 years, ${ }^{7,8}$ there is still some controversy in relation to their structures, stability, biosynthesis and ecological functions. In this review, a critical evaluation of existing data on spinochrome structures,

${ }^{a}$ Department of Food Science, University of Otago, PO Box 56, Dunedin 9054, New Zealand

${ }^{b}$ Laboratory of the Chemistry of Natural Quinonoid Compounds, G.B. Elyakov Pacific Institute of Bioorganic Chemistry, Far Eastern Branch of Russian Academy of Sciences, Prospect 100 let Vladivostoku 159/2, 690022, Vladivostok, Russia. E-mail: mischenkonp@mail.ru

'Department of Biochemistry, University of Otago, PO Box 56, Dunedin 9054, New Zealand

${ }^{d}$ Department of Microbiology and Immunology, University of Otago, PO Box 56, Dunedin 9054, New Zealand biosynthesis, distribution, isolation and identification techniques, stability, synthesis and biomedical applications is presented.

\section{The structure and nomenclature of spinochromes}

Spinochromes are polyhydroxylated derivatives of either juglone (5-hydroxy-1,4-naphthoquinone) or naphthazarin $(5,8$ dihydroxy-1,4-naphthoquinone) that are substituted with various functional groups such as ethyl, acetyl, methoxyl, and amino groups. The first discovered compound of this class, a red pigment in the perivisceral (coelomic) fluid of Echinus esculentus was called 'echinochrome', without knowing its chemical composition. 'The structure of this 'echinochrome' was established later by Kuhn and Wallenfels (1940) ${ }^{\mathbf{1 0}}$ as 6-ethyl2,3,7-trihydroxynaphthazarin and it was named echinochrome A (Table 1, structure 1). Subsequently, a number of pigment compounds were isolated from the shells and spines of various sea urchin species, which were named spinochromes to distinguish them from the echinochrome $\mathrm{A}$ found in the coelomic fluid, ovaries and internal organs of sea urchins. ${ }^{7}$ However, it was found later that echinochrome $\mathrm{A}$ is also a major pigment component of the shells and spines of many sea urchins, so it was assigned to the class of spinochromes, but the original name echinochrome has remained. ${ }^{\mathbf{1 8}}$

New spinochromes were named based on the first letter of the name of the sea urchin species that they were isolated from, as for example, spinochrome $\mathrm{P}$ was first isolated from 

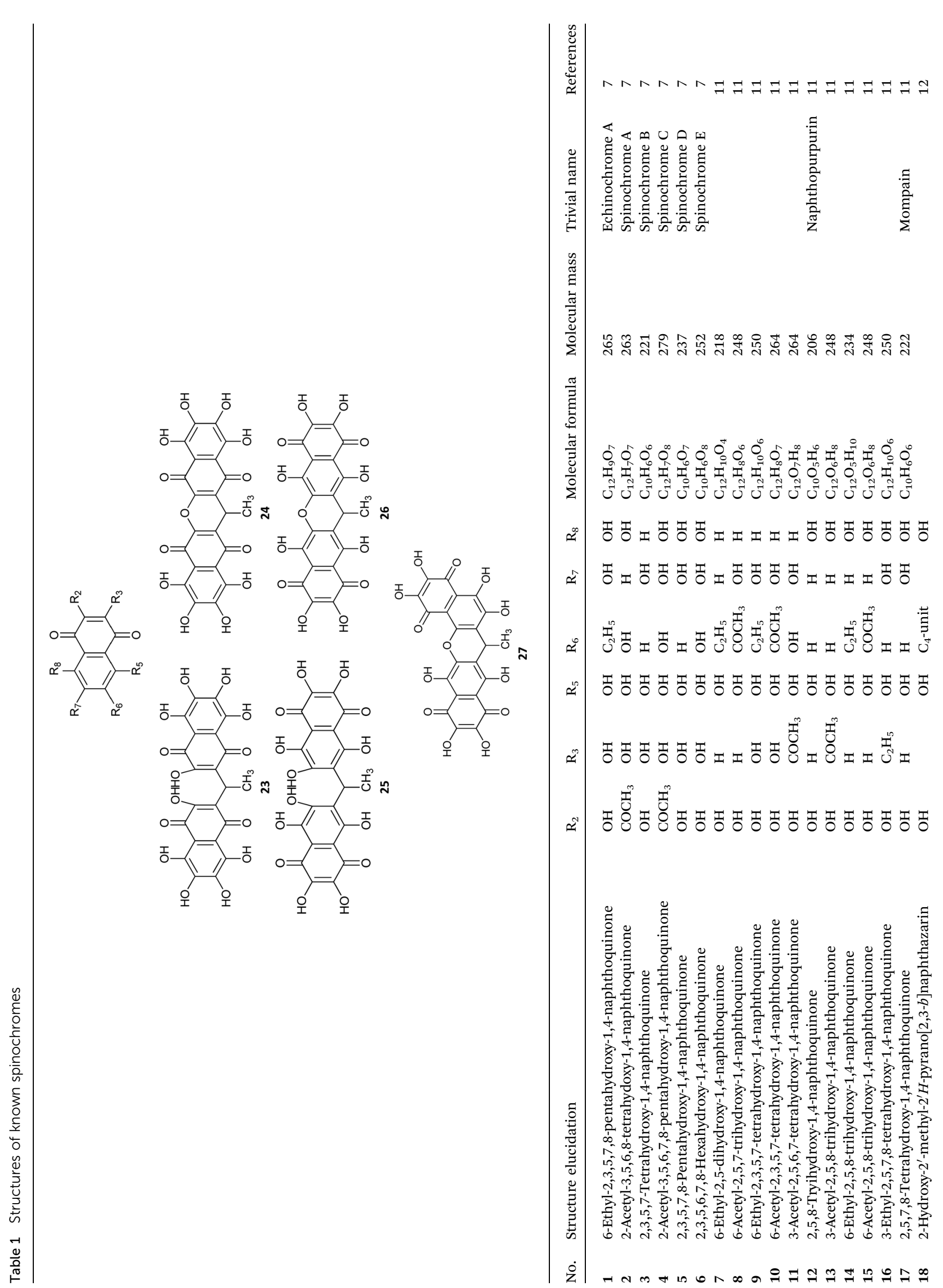

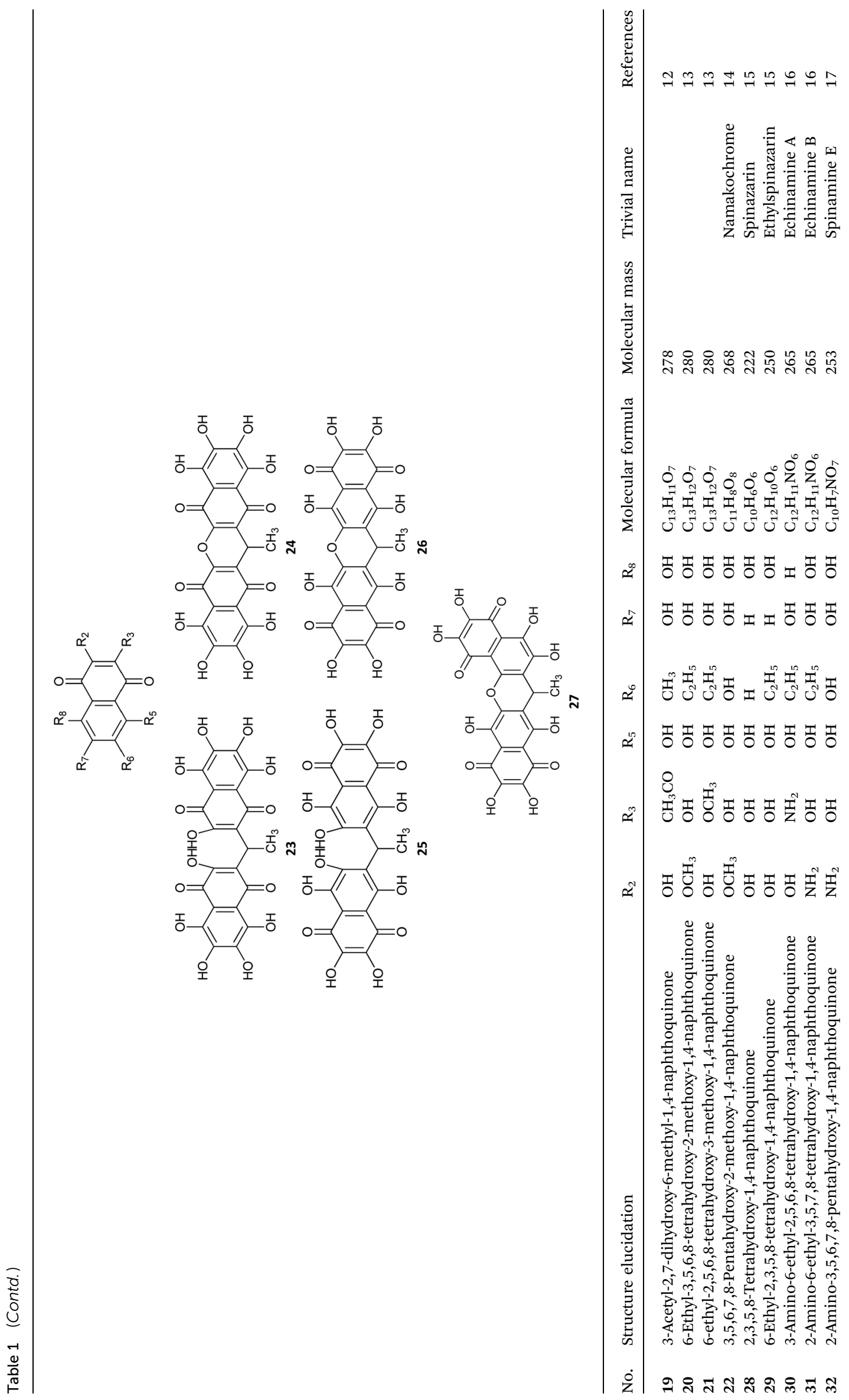
Paracentrotus lividus. Spinochromes extracted from several sea urchin species have been named as spinochromes A, F, C, M and $\mathrm{H}$ as well as others that can be found in the literature. ${ }^{18} \mathrm{It}$ was later shown that many quinones previously designated with different letters are in fact the same compound. Goodwin and Srisukh (1950) ${ }^{19}$ revised the nomenclature of all spinochromes known at that time and proposed naming them in alphabetic order, as spinochromes A (2), B (3), C (4), D (5) and E (6), as indicated for the structures summarised in Table 1 . Aside from the original naming of these six most commonly found spinochromes, polyhydroxylated naphthoquinone (PHNQ) pigments are now generally named as substituted juglones or naphthazarins. ${ }^{18}$

Anderson et al. (1969) ${ }^{7}$ summarized the data on the distribution of spinochromes in almost 60 species of sea urchins and established that echinochrome A and the spinochromes A-E are the most common pigments found in sea urchins. The composition of different PHNQs is specific for different sea urchin species, and in addition to the six most common spinochromes, some species may also contain specific structural analogues. For example, for the sea urchins Echinotrix calamaris and Echinothrix diadema from the Hawaiian coast, nine quinonoid pigments (7-16) that are derivatives of either juglone or naphthazarin were isolated along with mompain (17), commonly found in fungi, ${ }^{20}$ and 2 -hydroxy-2'-methyl-2' $H$-pyrano[3,2-b]-naphthazarin (18), the only spinochrome known to have a $\mathrm{C} 4$ unit attached to the naphthoquinone ring structure. ${ }^{\mathbf{1 1}}$ The compound 3-acetyl-2,7-dihydroxy-6-methylnaphthazarin (19) was first isolated from the shells and spines of the sea urchin Strongylocentrotus nudus (now known as Mesocentrotus nudus). ${ }^{21}$

Two monomethyl ethers of echinochrome A $(\mathbf{2 0}, \mathbf{2 1})$ have been isolated from the tropical sea urchin Diadema antillarum. ${ }^{\mathbf{1 3}}$ Methylated spinochromes previously had only been isolated from other echinoderms, such as asteroids, holothuroids and ophiuroids. ${ }^{13}$ Subsequently, these compounds were also found in other Diadema species such as D. setosum and D. savignyi ${ }^{22}$ and very recently in the north pacific sea urchin Strongylocentrotus droebachiensis. ${ }^{23}$ Furthermore, in S. droebachiensis and its related species S. polyacanthus, Vasileva et al. $(2017)^{23}$ reported two novel compounds by using HPLC-DAD-MS: monoand dimethoxy derivatives of spinochrome $\mathrm{E}$ (22) that have not been found in other sea urchins, reported previously only in asteroids and holothuroids.

From heart urchin Spatangus purpureus, the first spinochrome dimers - ethylidene-3,3'-bis(2,6,7-trihydroxynaphthazarin) (23) and its anhydro derivative (24) were isolated. ${ }^{\mathbf{1 3}}$ Later, Utkina et al. $(1976)^{24}$ working with Strongylocentrotus intermedius and Kol'tsova et al. $(1978)^{25}$ working with S. droebachiensis, isolated binaphthoquinones with the same molecular mass. Based on different melting point and detailed ${ }^{13} \mathrm{C}$ NMR spectra analysis it was established that the ethylidene bridge binds the phenyl but not quinonoid rings of the two naphthazarin fragments of the isolated binaphthoquinones, so their structures were elucidated as ethylidene-6,6'-bis(2,3,7trihydroxynaphthazarin) (25) and 7,7'-anhydroethylidene-6,6'bis(2,3,7-trihydroxynaphthazarin) (26). Recently, binaphthoquin- ones with characteristics similar to compounds 23 and 25 and its anhydrous derivative with characteristics similar to compounds $\mathbf{2 4}$ and 26 were isolated from the tropical sea urchin Astropyga radiata, and their structures were analysed using 2D-NMR procedures that were not available earlier. ${ }^{26}$ It was established that these compounds predominantly exist as ethylidene-3,3'-bis(2,6,7trihydroxynaphthazarin) (23) and 7,7'-anhydroethylidene-6,6'bis(2,3,7-trihydroxynaphthazarin) (26), respectively. ${ }^{26}$ In addition to these two binaphthoquinones from sand dollar Scaphechinus mirabilis, the isomer of $\mathbf{2 6}$ was isolated, which was the first unsymmetrical binaphthoquinone mirabiquinone $\left(7,5^{\prime}\right.$-anhydroethylidene-6,6'-bis(2,3,7-trihydroxynaphthazarin) (27). ${ }^{27}$

Four novel compounds, spinazarin (28) and ethylspinazarin (29) that previously were not considered to be natural pigments, and echinamines A (30) and B (31), the first representatives of aminated spinochromes, were all isolated from $S$. mirabilis. ${ }^{15,16}$ A Chinese group using UPLC identified two new aminated quinonoid pigments, aminopentahydroxynaphthoquinone and acetylaminotrihydroxynaphthoquinone, in the sea urchin Mesocentrotus nudus. ${ }^{5}$ Later, the aminopentahydroxynaphthoquinone was isolated from the sea urchins M. nudus and Strongylocentrotus pallidus, and the structure of the compound was elucidated to be 2-amino3,5,6,7,8-pentahydroxy-1,4-naphthoquinone using $1 \mathrm{D}{ }^{1} \mathrm{H}$ - and ${ }^{13} \mathrm{C}-\mathrm{NMR}$ and $2 \mathrm{D}$ NMR procedures, and was named spinamine $\mathrm{E}$ (32). ${ }^{17}$

Powell et al. $(2014)^{28}$ using LC-MS discovered sulphated spinochromes B and $\mathrm{E}$ and their structures were deduced based on the measurement of their accurate masses. Sulphated spinochromes had not been reported previously in sea urchins and the role of sulphated PHNQ pigments is still unknown. Further work is required to confirm their structures and characteristics.

\section{Biosynthesis of spinochromes}

In contrast to plant and fungal quinones, biogenesis of animalderived naphthoquinones is not well investigated. The pioneer study of spinochrome biosynthesis was performed by Salaque et al. (1967) $)^{29}$ using the adult Arbacia pustulosa sea urchin. In the study of Salaque et al., sea urchins were divided into three treatment groups and each group was injected in the coelomic cavity with either $\left[2-{ }^{14} \mathrm{C}\right]$-acetate, $\mathrm{L}-\left[\right.$ methyl $\left.-{ }^{14} \mathrm{C}\right]$-methionine or $\left[3^{-14} \mathrm{C}\right]$-propionate. Ten days after injection, the sea urchins were sacrificed, and their shells and internal organs were extracted with different solvents and the radioactivity in their biological materials was determined. The highest level of radioactivity was found to be incorporated in echinochrome $\mathrm{A}$ following $\left[2-{ }^{14} \mathrm{C}\right]$-acetate treatment, and the radioactivity level remained constant after three recrystallization steps to exclude contaminants and impurities. Interestingly, the radioactivity of the side chain of echinochrome A was relatively low, so it was suggested that biogenesis may proceed in two stages - first, cyclisation of a single polyketide derived from five acetate residues and then the introduction of the side chain. This was in agreement with the observation that spinochromes both with and without a two-carbon side chain (either ethyl or acetyl) frequently occur together in the same animal. Thus, Salaque 


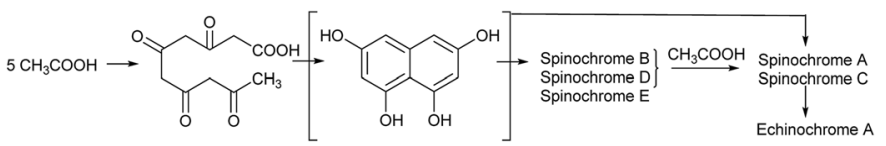

Fig. 1 Polyketide pathway of spinochrome biogenesis.

et al. (1967) established the de novo synthesis of echinochrome A in sea urchins and suggested a common scheme for spinochrome biogenesis (Fig. 1). Nevertheless, the authors did not exclude the participation of microbial flora in the biosynthesis process.

Another argument supporting the polyketide origin of spinochromes relates to the meta-positions of hydroxyls in spinochromes A and B, and that all alkylated naphthoquinones of sea urchins (as can be seen from Table 1) have a $-\mathrm{COCH}_{3}$ or $-\mathrm{CH}_{2}-$ $\mathrm{CH}_{3}$ side chain. However, no naphthoquinones having a methyl side-chain that is characteristic for plant quinones biosynthesized by other pathways (Table 1) are known. These features seem significant and are consistent with the polyketide hypothesis of biosynthesis formulated above.

To achieve polyketide biosynthesis in sea urchins, there is a need for specific enzymes. Polyketide synthases (PKS) are a large family of multifunctional enzymes. The PKSs catalyze the biosynthesis of different groups of compounds, which are mainly produced by bacteria, fungi, and plants. Animal PKS genes were first discovered in sea urchins, indicating that spinochrome pigments are produced by sea urchins with their own enzymes and likely not involving endosymbionts. ${ }^{30}$

Ageenko et al. (2011) ${ }^{31}$ analyzed PKS and sulfotransferase (sult) gene expression in embryos and larvae of the sea urchin Strongylocentrotus intermedius at various stages of development and in specific tissues of the adults. The authors found that addition of shikimic acid to zygotes and embryos increased the expression of the PKS and sult genes. Based on this, the authors suggested that spinochromes are generated after a series of enzymatic, oxidative, and photochemical reactions from shikimic acid, like that occurring for the formation of the plant quinone chimaphilin. However, animals (Metazoa), including sea urchins, do not have a shikimate pathway, ${ }^{32}$ since proteinogenic aromatic amino acids and other necessary products of the shikimate pathway are obtained in sufficient quantities from food (or from symbionts), it would appear that animals have not had a need to evolve de novo biosynthesis of shikimate derivatives.

\section{Functions of spinochromes}

Despite spinochromes having been studied for quite a long time, it is still not known what functions they perform in sea urchins. Since secondary metabolites are often considered to play a protective role against bacteria, fungi, amoebae in plants, insects, and large animals ${ }^{33}$ spinochromes might be utilized by sea urchins for protection. It has been shown that sea urchin extracts and individual spinochromes exhibit antimicrobial, ${ }^{34}$ antibacterial ${ }^{35-38}$ and anti-algal ${ }^{39}$ activities. Considering that quinonoid pigments are located in the shell of sea urchins, it seems logical that they may be intended for protection from various pathogens. ${ }^{34-39}$ Early suggestions that spinochromes have a respiratory function could not be confirmed, ${ }^{40,41}$ and the participation of spinochromes in photoreception remains an open question..$^{18}$ The presence of pigment granules with an unknown composition in coelomic fluid, ${ }^{42}$ eggs $^{43}$ and embryos ${ }^{44}$ of sea urchins has also been reported. It is believed that the pigment granules of the coelomic fluid, the red spherulocytes, might be involved in the immune response of sea urchins. Many studies have shown that the number of red spherulocytes significantly increases with stress in sea urchins, such as stress caused by a change in the temperature of the water or its salinity ${ }^{45,46}$ hypoxic conditions,${ }^{47}$ or wounding of the shell. ${ }^{48}$ The role of the pigment inclusions in the jelly coat of eggs of some sea urchin species and in embryos is still unclear.

\section{Distribution of spinochromes}

Currently there are slightly more than 1000 extant sea urchin species known from the oceans of the world, including the Arctic and Antarctic seas. ${ }^{49}$ However, only a very small number of investigations have been performed on spinochromes in these species to date. A search of the literature indicates that only around 70 species from the class Echinoidea have been investigated by marine natural product chemists. Anderson et al. (1969) ${ }^{7}$ conducted a large-scale study of the distribution of spinochromes in the shells of almost 60 species of regular (spherical) and irregular (flat and heart shaped) sea urchins. The data were summarised in a review and in a monography reported by his co-worker Thomson., ${ }^{718}$ The present review will focus on the distribution of spinochromes in sea urchins that have not been mentioned by Anderson et al. ${ }^{7}$ and Thomson ${ }^{18}$ and highlight the differences in pigment composition that have been reported more recently (Table 2).

\subsection{Regular sea urchins}

5.1.1 Family Strongylocentrotidae. Sea urchins of the genus Strongylocentrotus are very similar in morphology, but they are widely distributed geographically ${ }^{53}$ and vary in spinochrome composition.

Strongylocentrotus intermedius. The sea urchin $S$. intermedius is a valuable fishery resource of the Sea of Japan because their edible gonads are considered as a high value product in Asia. ${ }^{51}$ The composition of $S$. intermedius quinonoid pigments was investigated by Utkina et al. (1976) ${ }^{24}$ who isolated spinochromes A, B, C, D and 6,6'-ethylidene-bis(2,3,7-trihydroxynaphthazarin). Later Li et al. $(2013)^{51}$ isolated spinochrome B from $S$. intermedius using macroporous resin extraction from a crude pigment extract. 
Table 2 Distribution of spinochromes in sea urchins

The six main spinochromes

Order, family, species

1

2

$3 \quad 4$

5

6 Others

Reference

Camarodonta; Echinometridae

Echinometra mathaei

Anthocidaris crassispina

(transferred to Heliocidaris crassispina)

Camarodonta; Parechinidae

Psammechinus miliaris

Camarodonta; Strongylocentrotidae

Mesocentrotus nudus

Strongylocentrotus droebachiensis

$\begin{array}{ll}+ & + \\ + & + \\ + & +\end{array}$

+
+
+

+
+
+

$\begin{array}{rr}- & 6 \\ +\quad- & 35 \\ - & 50\end{array}$

$+\quad+\quad+$

$+$

Sulphate derivatives of $\mathbf{3}$ and $\mathbf{6 , 3 2}$

28

$+\quad+\quad+\quad+$

$+\quad 19$

21

+ Acetylaminotrihydroxynaphthoquinone,

17,32

$+\quad+\quad+\quad+\quad 32$

$+\quad 25,26$

25, 26, spinochrome dimers with

molecular masses 536 and 484

Strongylocentrotus intermedius

Strongylocentrotus pallidus Strongylocentrotus polyacanthus Camarodonta; Toxopneustidae Toxopneustes pileolus

Tripneustes gratilla

Clypeasteroida; Echinarachniidae

Echinarachnius parma

Clypeasteroida; Scutellidae

Scaphechinus mirabilis

Diadematoida; Diadematidae

Astropyga radiata

Spatangoida; Schizasteridae

Brisaster latifrons

Stomopneustoida; Glyptocidaridae

Glyptocidaris crenularis

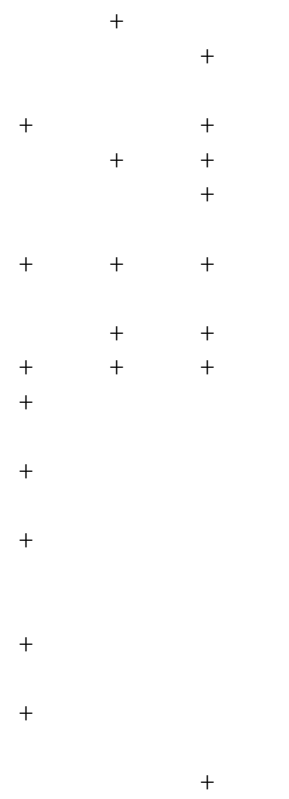

$+$

Strongylocentrotus droebachiensis. S. droebachiensis is commonly known as the green sea urchin because of its characteristic green colour. It is commonly found in northern waters all around the world including in both the Pacific and Atlantic oceans. $S$. droebachiensis from the Bering sea was investigated ${ }^{25}$ and spinochromes A, C, D, and E and anhydroethylidene-3,3'-bis-(2,6,7-trihydroxynaphthazarin) (now revised to anhydroethylidene-6,6'-bis-(2,3,7-trihydroxynaphthazarin)) were isolated from the shells and spines. Shikov et al. $(2011)^{4}$ using HPLC-DAD-MS detected spinochromes D and B, two binaphthoquinones with a molecular mass of 484, a binaphthoquinone with a molecular mass of 502, and an unidentified spinochrome dimer with a molecular mass of 536 in $S$. droebachiensis from the Barents Sea.

S. droebachiensis collected from the Barents Sea, the Bering Sea and in the Sea of Okhotsk had different spinochrome compositions. ${ }^{23}$ Even $S$. droebachiensis collected in the Sea of Okhotsk, but from different depths, were found to vary in pigment composition. ${ }^{23}$ Samples from a greater depth contained echinochrome A and 7,7'-anhydroethylidene-6,6'- bis(2,3,7-trihydroxynaphthazarin) as the main compounds, along with spinochrome E, its monomethyl ether namakochrome, spinochrome $\mathrm{D}$, mirabiquinone and two echinochrome A monomethyl ethers. In contrast, the samples collected from shallow waters contained 7,7'-anhydroethylidene-6,6'-bis(2,3,7-trihydroxynaphthazarin) as the main compound and only trace quantities of echinochrome A. ${ }^{23}$ The samples collected from shallow waters also contained ethylidene-3,3'-bis(2,6,7-trihydroxynaphthazarin), mirabiquinone, spinochromes E, D, and B, and an unidentified pigment with $m / z[\mathrm{M}-\mathrm{H}]^{-}$of 483 , which was assumed to be the same pigment detected by Shikov et al. ${ }^{4}$ Collectively, these results suggest that the composition of the secondary metabolites of marine organisms may significantly differ due to geographic, seasonal, gender and other variations. ${ }^{23}$

Strongylocentrotus pallidus. This species is a subtidal species found at depths of up to $1600 \mathrm{~m}$. It inhabits rocky areas, and it is found in the Pacific from the Kamchatka Peninsula to northern Alaska and Oregon, as well as in the North Atlantic. These sea urchins were difficult to harvest, and for a long time, they were confused with $S$. droebachiensis. Therefore, the chemical 
composition of this species was only studied recently. Only spinochrome E and spinamine $\mathrm{E}$ were identified in shell and spine extracts of $S$. pallidus. ${ }^{23}$

Strongylocentrotus polyacanthus. S. polyacanthus is abundant among the Aleutian Islands, in the Bering Sea, and along the coasts of the Kamchatka Peninsula, Korea, and Japan. The spinochrome composition of $S$. polyacanthus was investigated recently for the first time in samples that were collected near the Kuril Islands chain. ${ }^{23}$ One $S$. polyacanthus sampling contained two main compounds, spinochrome E and echinochrome A, and in addition, three minor spinochromes, spinamine $\mathrm{E}$, and spinochromes $\mathrm{C}$ and A were found. However, in another $S$. polyacanthus sampling, the main compounds found were spinochromes $\mathrm{E}$ and $\mathrm{A}$, and they were present in a different ratio compared to a previous sample. Spinochrome $\mathrm{C}$ was absent, but spinamine $\mathrm{E}$, spinochromes $\mathrm{D}$ and $\mathrm{B}$, and a dimethoxy derivative of spinochrome E were present. Again, this indicated that the location of sea urchins can affect the spinochrome composition and consequently the biological activity of extracts obtained from the sea urchins.

Mesocentrotus nudus. Sea urchins of the species M. nudus belong to the family Strongylocentrotidae that previously were considered to belong to the genus Strongylocentrotus but were transferred to the genus Mesocentrotus based on the results of DNA-DNA hybridization and comparative morphology. ${ }^{54} \mathrm{M}$. nudus is a dominant sea-urchin species in the northwest Pacific and is found on intertidal and subtidal rocky sea bottoms. ${ }^{55}$ The quinonoid pigments of the shells and spines of $M$. nudus consist mainly of spinochromes $\mathrm{A}, \mathrm{B}$, and $\mathrm{C}$ and echinochrome $\mathrm{A}$. Although 3-acetyl-2,7-dihydroxy-6-methylnaphthazarin was isolated from the test and spines of $M$. nudus in $1977,{ }^{21}$ it was obtained after vacuum sublimation and has not been detected since then in extracts of $M$. nudus, so this pigment may not be of natural origin. In 2012 two new aminated quinonoid pigments (aminopentahydroxynaphthoquinone and acetylaminotrihydroxynaphthoquinone) were found in M. nudus by using UPLC .5 Aminopentahydroxynaphthoquinone was isolated by Vasileva et al. $(2016)^{17}$ and its structure was established as 2-amino3,5,6,7,8-pentahydroxy-1,4-naphthoquinone using 1D- and 2DNMR and was named spinamine E.

\subsubsection{Family Toxopneustidae}

Toxopneustes pileolus. T. pileolus, commonly known as the flower urchin, is a widespread and commonly encountered species of sea urchin in the Indo-West Pacific region. Unlike Strongylocentrotus, it is considered highly dangerous, as it is capable of delivering extremely painful and medically serious stings when touched. Many studies of the sea urchin T. pileolus have been dedicated to investigating toxic compounds released from its various organs, especially the pedicellariae. Kol'tsova and Krasovskaya (2009) ${ }^{52}$ observed only spinochrome A in the pedicellariae of this species that was collected near Nha Trang Bay in the South China Sea, while spinochrome A, B and C were present in the shells and spines at different ratios in relation to the colour intensity of shells. T. pileolus collected from the coral reef at Toliara, Madagascar, contained echinochrome A and spinochromes A-E. ${ }^{35}$
Tripneustes gratilla. T. gratilla, also known as the collector urchin, inhabits the waters of the Indo-Pacific, Hawaii, the Red Sea, and the Bahamas. Early studies on this species isolated only spinochrome A and mompain from the shells of $T$. gratilla from Hawaii, ${ }^{20}$ but recent studies on $T$. gratilla harvested near Madagascar reported a different pigment pattern that contained spinochromes $\mathrm{D}$ and $\mathrm{E}$ and echinochrome $\mathrm{A} \cdot{ }^{35}$

\subsubsection{Family Glyptocidaridae}

Glyptocidaris crenularis. The sea urchin G. crenularis is the only species known of the Glyptocidaris genus. It lives in the waters of the Pacific Ocean and is distributed widely in the Sea of Japan and the Yellow Sea of China. ${ }^{56}$ Spinochrome E, spinochrome D and spinochrome B were tentatively identified in the pigment extract prepared from $G$. crenularis..$^{51}$

\subsubsection{Family Parechinidae}

Psammechinus miliaris. P. miliaris, also known as the green sea urchin or shore sea urchin, is found in shallow waters of the Eastern Atlantic Ocean and the North Sea. The gonads of $P$. miliaris are eaten particularly in Mediterranean cuisine. ${ }^{57}$ LCMS analysis of shell and spine extracts indicated the presence of spinochromes A, B, C and E, spinamine $\mathrm{E}$ and echinochrome A, but also revealed the existence of other related components with mass spectral properties consistent with sulphated or phosphorylated spinochromes B and E. ${ }^{28}$

\subsubsection{Family Echinometridae}

Heliocidaris crassispina (earlier called Anthocidaris crassispina). $H$. crassispina is a species containing edible gonads that is found in the Pacific coastal regions around Ibaragi, and in the southern Japan Sea around Akita, and around Taiwan and the Southeast coast of China. ${ }^{58}$ Kuwahara et al. (2009) ${ }^{50}$ using LCMS, identified spinochromes A, B and C and echinochrome A in the shells of $H$. crassispina, in agreement with an earlier report. ${ }^{7}$

Echinometra mathaei. E. mathaei, the burrowing urchin, is found in the shallow waters of the Indo-Pacific region. Its distribution range extends from Madagascar on the East African coast to the Red Sea and to Hawaii. The main pigments found in this species are the spinochromes A, B and C, ${ }^{7}$ and recently the presence of echinochrome $\mathrm{A}^{6}$ and spinochrome $\mathrm{E}^{35}$ were reported.

\subsubsection{Family Diadematidae}

Astropyga radiata. A. radiata, known either as the red urchin, fire urchin, false fire urchin or blue-spotted urchin, is a large species with long spines and is found in the tropical Indo-Pacific region. ${ }^{49}$ Spinochrome $\mathrm{E}$ and $\mathrm{D}$, echinochrome A, binaphthoquinones ethylidene-3,3-bis(2,6,7trihydroxynaphthazarin) and 7,7'-anhydroethylidene-6,6'-bis(2,3,7-trihydroxynaphthazarin) have been reported to be present in this species. ${ }^{23,27}$

\subsection{Irregular sea urchins}

\subsubsection{Family Echinarachniidae}

Echinarachnius parma. E. parma is a species of sand dollar native to the Northern Hemisphere. It is found in the North Pacific, on the North American east coast as well as in Alaska, Siberia, British Columbia, and Japan, and in the Northwest 
Atlantic. It inhabits isolated areas on sandy bottoms below the low tide level and down to a depth of $1500 \mathrm{~m} \cdot{ }^{49}$ Identification of PHNQ pigments from E. parma was performed for the first time recently. ${ }^{23}$ It was found that E. parma had three main components: spinochrome D, 7,7'-anhydroethylidene-6,6'-bis(2,3,7trihydroxynaphthazarin) and echinochrome $\mathrm{A}$, as well as spinochrome E, mirabiquinone and ethylidene-3,3'-bis(2,6,7-trihydroxynaphthazarin), and two aminated derivatives, echinamines A and B, were detected in trace quantities.

\subsubsection{Family Scutellidae}

Scaphechinus mirabilis. The sand dollar $S$. mirabilis is distributed around the Commander Islands, on the east coast of the Kamchatka Peninsula, the Japanese Islands and in Peter the Great Bay (Sea of Japan) on soft sea floors. ${ }^{59}$ In the last decade, several novel spinochromes have been discovered in S. mirabilis. Echinamines A and B, which were the first examples of natural polyhydroxynaphthazarins containing a primary amino group, were discovered in $2005 .{ }^{16}$ After that spinazarin $(2,3,5,8$ tetrahydroxy-1,4-naphthoquinone) and ethylspinazarin(6-ethyl2,3,5,8-tetrahydroxy-1,4-naphthoquinone) were first isolated from $S$. mirabilis. ${ }^{15}$ Mirabiquinone, the first unsymmetrical binaphthoquinone, was also discovered in this species. ${ }^{27}$

\subsubsection{Family Schizasteridae}

Brisaster latifrons. B. latifrons is a heart shaped urchin occurring on sandy sea floors at a depth of up to $1820 \mathrm{~m}$ from the west coast of Central America, to the Galápagos Islands, and to the west coast of México, and in the Gulf of California. ${ }^{60}$ In B. latifrons extracts, echinochrome A was found to be the main compound along with three other minor components, spinochromes $\mathrm{E}$ and $\mathrm{D}$, and $7,7^{\prime}$-anhydroethylidene-6, $6^{\prime}$ bis(2,3,7-trihydroxynaphthazarin) that were identified recently. ${ }^{23}$

From the reported data on spinochrome composition in different sea urchins, it can be seen that spinochrome pigment compositions are not always species-specific. In addition, sea urchins from one genus may have different compositions of spinochromes, as for example in the case of the Strongylocentrotus sea urchins (Table 2). Nevertheless, it is possible to trace certain regularities. For example, both sand dollars from the order Clypeasteroida contain echinochrome A and binaphthoquinones (23, 26 and 27), and the majority of tropical sea urchins contain echinochrome $\mathrm{A}$ as a main pigment.

Interestingly, in many cases recent spinochrome composition determination differs from that published previously. For example, earlier findings by Anderson and co-authors did not describe echinochrome A as being present in E. mathaei, $H$. crassispina, $P$. milliaris, $T$. gratilla ${ }^{7}$ but recent reports have found this compound in all of these species. ${ }^{6,28,35,61}$ It was likely more challenging to identify spinochromes earlier because of a lack of modern instrumentation such as LC-MS and high-resolution NMR. It is also well known that the composition of secondary metabolites in marine organisms may differ significantly due to geographic, ecological, seasonal, gender and other variables. Therefore, it is very likely that differences in spinochrome compositions in sea urchins of the same species may be due to these variables. For example, $S$. droebachiensis collected from the Barents Sea, ${ }^{4}$ the Bering Sea ${ }^{21}$ and the Sea of Okhotsk ${ }^{23}$ contained different compositions of spinochrome pigments. Even $S$. droebachiensis collected in the Sea of Okhotsk, but from different depths, had variations in pigment composition and content. Since several sea urchin species such as $S$. mirabilis and $S$. droebachiensis are harvested commercially and the pigments extracted are being used in biomedical preparations, it is important to take into account geographic, environmental and other factors and provide information on the composition of quinonoid pigments before processing raw materials.

\section{Extraction, fractionation and identification of spinochromes}

Considering that sea urchin quinones most commonly occur in shells and spines in pigment granules associated with the $\mathrm{Ca}^{2+}$ and $\mathrm{Mg}^{2+}$ based mineral phase and proteins, chemical reagents need to be used to release the pigments from this material. The general scheme of spinochrome pigment isolation is to treat sea urchin skeleton with an acidic solution to solubilise the mineral structure, followed by extraction of the pigments from the acidic solution with one or more organic solvents and then fractionation of the crude pigment mixture by chromatography (Fig. 2).

Hydrochloric acid is commonly used for dissolving the shell and spine mineral structure and the obtained solution is exhaustively extracted with diethyl ether. ${ }^{\mathbf{4}, \mathbf{1 3 , 3 5 , 5 0 , 6 2 , 6 3}}$ The use of other organic solvents has been reported, including chloroform, ${ }^{23}$ ethyl acetate ${ }^{28}$ and butanol ${ }^{52}$ that have been used to extract the pigments from the acid solubilised solution. Zhou et al. ${ }^{5}$ and $\mathrm{Li}$ et al. ${ }^{51}$ noted that the reaction of hydrochloric acid with sea urchin skeleton generated bubbles of carbon dioxide which they said affected the subsequent extraction, so they reported the use of an $\mathrm{HCl}$-ethanol-aqueous solution with further fractionation of the pigment extract on NKA-9 macroporous resin. Mishchenko and co-workers ${ }^{16,17,23,27}$ used ethanol

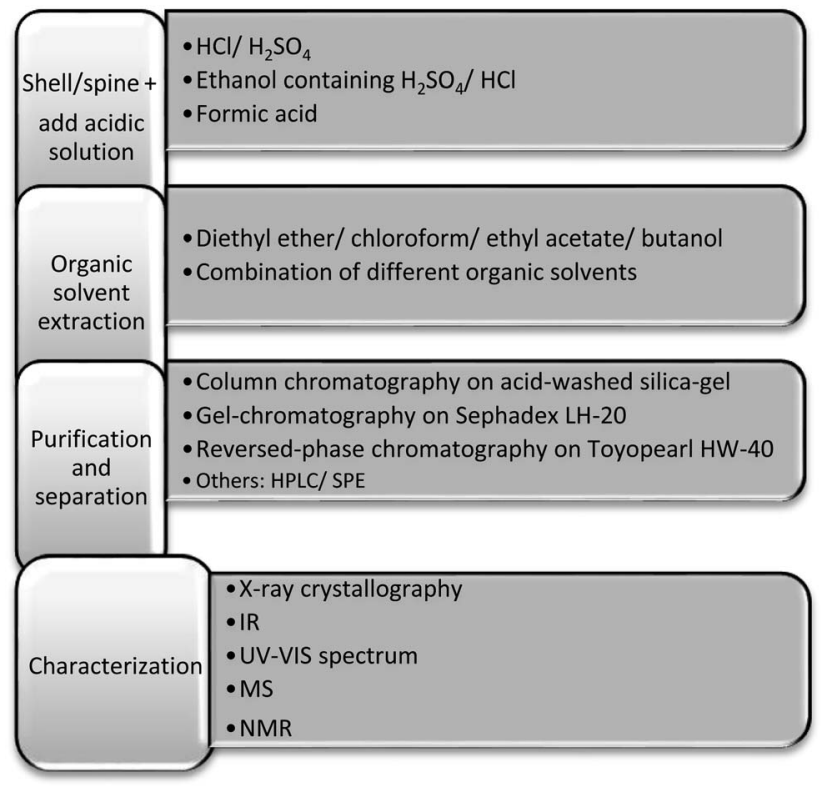

Fig. 2 Summary of extraction, purification and identification methods for spinochrome pigments from sea urchin shells and spines. 
containing $10 \% \mathrm{H}_{2} \mathrm{SO}_{4}$ for spinochrome extraction which forms water-insoluble $\mathrm{CaSO}_{4}$, that in contrast to the water-soluble $\mathrm{CaCl}_{2}$ produced by hydrochloric acid solubilisation, can be easily removed by filtration or centrifugation. Following vacuum concentration, the ethanolic extract was then partitioned between water and either chloroform or ethyl acetate. ${ }^{16,17,23,27}$ Some investigators have used organic acids for the release of pigments from the shells and spines. For example, Powell et al. ${ }^{28}$ suggested formic acid for spinochrome extraction because carbon dioxide was not generated as a result of the reaction between the acid and calcium carbonate in the shell. Further pigment mixture fractionation was performed using solid phase extraction (SPE) GIGA C18E units that allowed a cleaner and better fractionation, but the authors noted a reduced yield of pigments due to a low desorption rate. ${ }^{28}$

Methods of purification and identification of spinochrome pigments are similar to those commonly used in natural product chemistry. Usually spinochromes are separated by column chromatography on acid-washed silica-gel, ${ }^{7,24,25,63}$ followed by gel-chromatography on Sephadex LH-20 ${ }^{62}$ or reversedphase chromatography on Toyopearl HW-40. ${ }^{16}$ To obtain more soluble forms of spinochromes for effective separation during subsequent chromatography, acetates, leuco-acetates and methyl ethers of hydroxyquinones can be prepared. Based on the assumption that spinochrome methyl ethers do not occur naturally, Thomson et al. ${ }^{18}$ methylated partially purified fractions, separated them and hydrolysed the isolated individual methyl ethers. However, as spinochrome methyl ethers have subsequently been found in several sea urchin species, ${ }^{7,22,23,25}$ this technique should be applied with careful consideration. ${ }^{18}$

Various colour reactions have been used for the identification of quinonoid pigments. ${ }^{18}$ Over the years, colour reactions and TLC-chromatography have been replaced with modern spectroscopic methods. A significant contribution to spectroscopic and mass-spectrometric studies of spinochrome pigments was made by a group of scientists under the supervision of Paul Scheuer from the University of Hawaii, who used ${ }^{1} \mathrm{H}$ NMR, UV and mass-spectrometry to study a large number of substituted naphthoquinones. ${ }^{\mathbf{1 1 , 6 4 , 6 5}}$

The absorption spectra of spinochromes are very characteristic and consist of a combined benzenoid and quinonoid band at $\lambda$ 254-290 nm, a small quinonoid band in the 310-480 nm region and a benzenoid multi-banded absorbance centred near $500 \mathrm{~nm} .{ }^{65}$ The presence of a methoxyl substituent in a 1,4naphthazarin molecule bathochromically shifts the absorbance band at $500 \mathrm{~nm}$ to $520-530 \mathrm{~nm} .^{23,66}$ The quinonoid electron transfer band and combined benzenoid and quinonoid band in the absorption spectrum of the aminated spinochromes are usually bathochromically shifted $15-20 \mathrm{~nm}$ compared with that of their hydroxylated analogues. ${ }^{16,17}$ Acetyl substituents contribute unambiguously to the absorption spectra of spinochromes, as for example, despite spinochromes A and $\mathrm{C}$ both having an acetyl substituent and differing only in one hydroxyl group, their absorption spectra are quite different. ${ }^{5,23}$

The quinonoid carbonyl frequencies of naphthazarins are useful diagnostic aids in the determination of their structures. ${ }^{18}$ The carbonyl absorption $\nu_{\mathrm{CO}}$ of naphthazarins occurs at 1590-
$1620 \mathrm{~cm}^{-1}$ and in the same interval $\mathrm{C}=\mathrm{O}$ and $\mathrm{C}=\mathrm{C}$ correlations are displayed as a mixed band. In the IR spectra of naphthazarins, there is a broad (2200-3400 $\mathrm{cm}^{-1}$ ) absorption band corresponding to $\alpha$ hydroxyls that form a strong intramolecular hydrogen bond with quinonoid carbonyls. Signals emanating from $\beta$ hydroxyl and amino substituents are located at frequencies in the range of $3310-3525 \mathrm{~cm}^{-1} .^{67}$

Moore et al. (1966) ${ }^{11}$ correlated the chemical shifts in ${ }^{1} \mathrm{H}$ NMR spectra of hydroxyl, acetyl, ethyl, methoxyl, and acetoxyl substituents and their influence upon each other at various naphthoquinone ring positions. It was established that $\alpha$ hydroxyl protons give singlet signals around $\delta_{\mathrm{H}}$ 11.5-13.3. Generally, signals of $\beta$ hydroxyl protons $\left(\delta_{\mathrm{H}} 6.4-10.5\right)$ and amino group protons $\left(\delta_{\mathrm{H}} 5.4-6.0\right)$ appear as broadened singlets. The chemical shift of the free proton in the naphthoquinone nucleus is around $\delta_{\mathrm{H}}$ 6.2-7.6. Ethyl substituents give two characteristic signals - a quartet of a methylene group $\left(\delta_{\mathrm{H}} 2.6-2.8\right)$ and a triplet of a methyl group $\left(\delta_{\mathrm{H}} 1.1-1.3\right)$. The singlet signal of an acetyl substituent has a $\delta_{\mathrm{H}}$ value around 2.5-2.9, and of a methoxyl, 3.9-4.2. Signals of the ethylidene bridge that connects naphthazarin moieties of bi-naphthoquinones are comprised of a quartet at $\delta_{\mathrm{H}}$ 4.4-4.8 and a doublet at $\delta_{\mathrm{H}}$ 1.4-1.6. Until recently ${ }^{13} \mathrm{C}$ NMR data was not available for the majority of spinochromes, but in last decade it has been reported for a number of these compounds. Chemical shifts associated with the nodal carbons C-9 and C-10 have $\delta_{\mathrm{C}}$ values around 101.8110.2. Signals of carbonyl carbons C-1 and C-4 appear at $\delta_{\mathrm{C}}$ 169.0-182.8. Carbons attached to hydroxyls have a $\delta_{\mathrm{C}}$ value of 135.8-160.6. The chemical shift of the carbon atom substituted with an aliphatic chain is around $\delta_{\mathrm{C}}$ 120.0-136.7. The methyl carbon of an ethyl substituent has a chemical shift at $\delta_{\mathrm{C}} 12.0-$ 13.5, and a methylene carbon at $\delta_{\mathrm{C}}$ 16.2-17.2. An ethylidene bridge exhibits a signal corresponding to a methyl carbon at $\delta_{\mathrm{C}}$ 17.6-22.7 and a methine carbon at $\delta_{\mathrm{C}} 23.1-27.7$. The signal of a methoxyl carbon has a chemical shift of around $\delta_{\mathrm{C}}$ 60.9-61.6.

In the last decade, a total chemical shift assignment based on 2D NMR experiments, specifically Heteronuclear Multiple Bond Correlation (HMBC), have been reported for a number of spinochrome pigments. Interpretation of HMBC data for spinochromes is usually complicated because the proton signals of $\beta$ hydroxyls are broad and give no sharp peaks. To date, for several spinochromes a total chemical shift assignment has been reported based on 2D NMR, including spinochrome $\mathrm{D},{ }^{26}$ echinochrome $\mathrm{A},{ }^{26}$ echinochrome A methyl ethers, ${ }^{23}$ echinamines $\mathrm{A}$ and $\mathrm{B},{ }^{\mathbf{1 6}}$ spinamine $\mathrm{E},{ }^{17}$ mirabiquinone,${ }^{27}$ ethylidene3,3'-bis(2,6,7-trihydroxynaphthazarin) and 7,7'-anhydro-ethylidene-6, $6^{\prime}$-bis(2,3,7-trihydroxynaphthazarin). ${ }^{26}$

Although X-ray crystallography has played a key role in determining the structure of numerous marine natural products for decades, there is only one report of a crystallographic study of sea urchin quinonoid pigments. ${ }^{68}$ This X-ray analysis examined the molecular structure of echinochrome and the stable complex formed during the extraction and purification of echinochrome using dioxane. ${ }^{68}$

The electron-impact-induced fragmentation characteristic for naphthoquinones is the loss of one or two molecules of carbon monoxide and elimination of an acetylenic fragment 
from the quinonoid ring. If the remaining fragment is hydroxylated, the breakdown is accompanied by a characteristic hydrogen rearrangement. ${ }^{69}$ Nowadays, the most common type of ionization for spinochrome identification is electrospray (ESI), under such conditions PHNQ form $[\mathrm{M}-\mathrm{H}]^{-}$ions easily. ${ }^{4,5,23,28,35,51}$ High resolution ESI mass spectrometry and MS/ MS techniques have been widely used., ${ }^{\mathbf{4 , 5 2 8 , 5 1}} \mathrm{MS} / \mathrm{MS}$ fragmentation involves either a loss of a molecule of water (M-18), or a molecule of carbon monoxide (M-28), or loss of both of them $(\mathrm{M}-46) .{ }^{28}$

Currently, HPLC coupled with UV and mass detection is being used as a rapid and accurate method for the identification of spinochrome pigments.

\section{Stability of spinochromes}

It is important to investigate the stability of spinochromes during isolation, separation and storage since it is critical for their functionality and application. However, there are only a few publications available on the stability of spinochromes under different chemical conditions. Zhou et al. ${ }^{\text {70 }}$ investigated the stability of spinochrome crude mixtures recovered from $S$. nudus shells. The authors found that pigments in aqueous solution were prone to degradation upon exposure to light and elevated temperature. Several factors such as exposure to alkaline conditions, oxidizing agents and some metal ions such as $\mathrm{Ca}^{2+}, \mathrm{Zn}^{2+}, \mathrm{Al}^{3+}$ and $\mathrm{Fe}^{3+}$ may increase the oxidation rate of the pigments. ${ }^{70,71}$ However, the authors used for their experiments a centrifuged aqueous solution of pigments, despite that most spinochromes are poorly soluble in water. Consequently, the characteristic naphthoquinone absorption band at $470 \mathrm{~nm}$ in the absorption spectra that they obtained was quite low in intensity, compared to other bands, that indicated the low purity of the spinochromes solutions. Hence, the conclusions made by the authors about the stability of spinochromes under different conditions requires careful consideration.

Shikov et al. ${ }^{72}$ evaluated the stability of individual pigments from $S$. droebachiensis, including spinochrome D, an unidentified spinochrome dimer and ethylidene 6,6'-bis(2,3,7trihydroxynaphthazarin). The dimeric pigments remained stable in strongly acidified ethanol solution at $\mathrm{pH}$ 1.6, and were prone to degradation with an increase in $\mathrm{pH}$ up to 6.0 , while spinochrome D was found to be stable up to $\mathrm{pH}$ 4.0. Vasileva and co-authors compared the stability of aminated spinochromes and their hydroxylated analogues in HEPES buffer at $\mathrm{pH}$ 7.5. ${ }^{17}$ Echinochrome A, echinamine A and echinamine B were found to be stable under these conditions, while spinochrome $\mathrm{E}$ and spinamine $\mathrm{E}$ were prone to degradation.

\section{Bioactivities of spinochromes}

Even though many investigations of the bioactivities of spinochromes have been conducted, most of these studies have been based on crude extracts and not on isolated spinochromes. In this review we will focus mainly on the bioactivities of individual pigments.

A number of investigations have reported that spinochromes are strong antioxidants that can block a number of free radical reactions, inhibit lipid peroxidation, and reduce and chelate metal ions..$^{\mathbf{5 6}, 28,50,51,73-76}$ The antioxidant effect in vitro of spinochromes depends on the number and mutual positions of the chemical substituents in the molecule. ${ }^{75,76}$ Substitution of hydroxyl groups with methoxyls in the naphthoquinone molecule leads to the loss of antioxidant activity. ${ }^{75}$ Recently it has been shown that aminated spinochromes are more potent antioxidants than their hydroxylated analogues. ${ }^{\mathbf{1 6 , 7 6}}$

Echinochrome A and the spinochromes A-E were studied for their antimicrobial activity by Stekhova et al. ${ }^{34}$ who reported that only echinochrome A exhibited some antimicrobial effect towards the fungi Saccharomyces carlsbergensis, Candida utilis and Trichophyton mentagrophytes, and towards Staphylococcus aureus bacteria. Unlike antioxidant activity, the antimicrobial effect of spinochromes did not depend on the location and number of hydroxyl groups in the naphthoquinone molecule, while the presence of the ethyl substituent considerably increased the antimicrobial activity. The antimicrobial activity of trimethyl esters of echinochrome A and spinochrome B was significantly higher than that of their hydroxylated analogues. ${ }^{34}$

Brasseur et al. $^{35}$ compared the antibacterial activity of crude extracts and individual spinochromes from four sea urchin species and demonstrated that the results of bioassays differ when using crude extracts compared to purified spinochromes. The echinochrome A/spinochrome $\mathrm{C}$ mixture and spinochrome $\mathrm{A}$ were active inhibitors against Shewanella oneidensis. Spinochromes B and E had variable inhibitor activity that was dependent on the bacterial strain. The authors showed spinochrome B and spinochrome $\mathrm{E}$ had high antibacterial activity against Escherichia coli, but they were less effective against other bacteria such as Bacillus subtilis and Vibrio aestuarianus. Brasseur et al. ${ }^{35}$ also showed that each isolated spinochrome significantly increased the TNF-alpha production by LPS-stimulated macrophages inducing a pro-inflammatory activity, and that all isolated spinochromes exhibited low cytotoxicity against HeLa cells.

Echinochrome A and spinochrome A appeared to be inhibitors of tyrosine hydroxylase and dopamine- $\beta$-hydroxylase, which are targets for treatment of hypotension and other conditions. ${ }^{77,78}$ However, the inhibition mechanism is still not well understood. The authors suggested that this might be due to formation of a complex through an enzyme-bound metal, or due to the non-enzymatic oxidation of the reducing cofactor and the resultant consumption of oxygen in the reaction mixture.

Until recently there was no information about bioactivities of binaphthoquinones from sea urchins except for antioxidant activity. ${ }^{27}$ Recently, an extract of Strongylocentrotus droebachiensis containing the spinochromes B and D and binaphthoquinones 25 and 26, was shown to exhibit a significant anti-allergic property. ${ }^{3}$ It reduced the histamine-induced contractions of isolated guinea pig ileum in a dose-dependent manner, had an inhibitory effect on a model of ocular allergic inflammation, and did not show any irritating effect in rabbits. ${ }^{3}$ Molecular docking of investigated compounds into an $\mathrm{H}_{1} \mathrm{R}$ receptor demonstrated that both monomer and dimer spinochromes bind successfully to the receptor active site, but monomers fit better. On the basis of this study a biomedical preparation is being developed in Russia with anti-inflammatory and anti-allergic effects. ${ }^{79}$ 


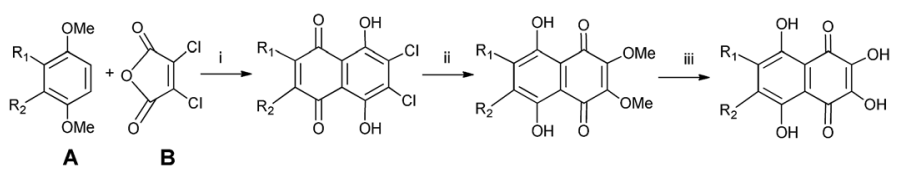

Scheme 1 Synthesis of spinochromes as described by Singh et al. ${ }^{90}$ Reagents and conditions: (i) $\mathrm{AlCl}_{3}-\mathrm{NaCl}, 195{ }^{\circ} \mathrm{C}$; (ii) $\mathrm{MeONa}, \mathrm{MeOH}$, reflux; (iii) conc. $\mathrm{HBr}$, reflux.

\section{Biomedical applications of echinochrome A}

Among all the known spinochromes, echinochrome A is the most well studied compound in terms of biomedical applications. The antioxidant pharmaceutical "Histochrome" was created on the basis of the pigment echinochrome A. ${ }^{73}$ This product was developed at the Laboratory of the Chemistry of Natural Quinonoid Compounds, Pacific Institute of Bioorganic Chemistry. ${ }^{73}$ It is a water-soluble sodium salt of echinochrome A, a pentahydroxyethylnaphthoquinone, dissolved in $0.9 \%$ sodium chloride solution. It is registered as a medicinal product authorized for use in the Russian Federation in the pharmacotherapeutic group "antioxidant agent". It is recommended as an antioxidant, cardioprotector and as an ophthalmic and antiarrhythmic medicine. ${ }^{73}$ Histochrome has been demonstrated to be an effective drug for the treatment of proliferative processes, degeneracies, and ophthalmic hemorrhages of various types. ${ }^{73,80,81}$ However, the effect of this medicine is very difficult to explain in terms of the antioxidant properties alone. The results of experimental investigation of echinochrome A both in vivo and in vitro showed that this compound has far more biological effect than that found for the Histochrome product, including anti-fibrosis, anti-diabetic, anti-allergic, anti-acetylcholinesterase, mitochondria-protective, gastroprotective and other effects. ${ }^{\mathbf{8 2 - 8 9}}$ Therefore, development of new formulations based on echinochrome directed to providing targeted and controlled release of this compound may expand the application of this compound.

\section{Synthesis}

As spinochromes have been shown to exhibit a wide range of bioactivities, several attempts have been made to develop synthesis pathways for effective production. ${ }^{\mathbf{9 0}-99}$ Synthesis of various spinochromes, including echinochrome A and spinochromes A, C, D, and E, were described five decades ago. ${ }^{65,90}$ The naphthoquinone skeleton of the target compound was constructed by condensing dihydroxy-3,4-dimethoxybenzene (A) with dichloromaleic anhydride (B) in an $\mathrm{AlCl}_{3}-\mathrm{NaCl}$ melt at $195{ }^{\circ} \mathrm{C}$. Then, the $\mathrm{Cl}$ atoms are replaced with $\mathrm{OMe}$ (ii) groups and demethylated with $\mathrm{HBr}$ (iii) according to the conditions given in Scheme 1.

However, this synthesis pathway was not suitable for preparative use due to cost. At present, the Anufriev group has developed pathways of preparative synthesis for almost all of the spinochromes. As starting compounds, commercially available 5,8-dihydroxy-1,4-naphthoquinone (naphthazarin) or 2,3-dichloronaphthazarin are usually used. Thus, echinochrome A, ${ }^{91}$ spinochrome C, ${ }^{92,93}$ spinochrome D, ${ }^{92}$ spinochrome $\mathrm{E},{ }^{94}$ echinamines $\mathrm{A}$ and $\mathrm{B},{ }^{95}$ spinamine $\mathrm{E},{ }^{96}$ and a pigment of Echinothrix diadema containing a pyran unit, 2-hydroxy-2'methyl-2' $H$-pyrano[3,2-b]-naphthazarin $(\mathbf{1 8})^{\mathbf{9 7}}$ have been synthesized.

Recently, a more simple and effective synthesis of spinochrome $\mathrm{E}$ has been described by Borisova and Anufriev. ${ }^{\mathbf{9 4}}$ Preparative synthesis starts with 2,3-dichloro-6,7diethoxynaphthazarin (C) with the simultaneous replacement of $\mathrm{Cl}$ atoms with hydroxyl and nitro groups followed by reduction of the latter, and subsequent removal of alkoxy groups

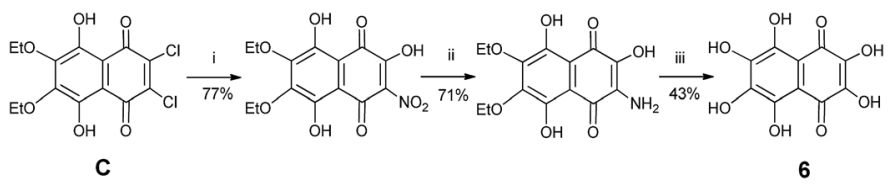

Scheme 2 Synthesis of spinochrome $\mathrm{E}(6)$ as described by Borisova and Anufriev. ${ }^{94}$ Reagents and conditions: (i) $(\mathrm{EtO}){ }_{3} \mathrm{CH}$, reflux; (ii) $\mathrm{NaNO}{ }_{2}$, EtOH, $\mathrm{H}_{2} \mathrm{O}$, heating; (iii) $\mathrm{Na}_{2} \mathrm{~S}_{2} \mathrm{O}_{4}, \mathrm{H}_{2} \mathrm{O}$; (iv) $\mathrm{AlCl}_{3}, \mathrm{PhNO}_{2}$, heating, then $5 \% \mathrm{HCl}$, heating.

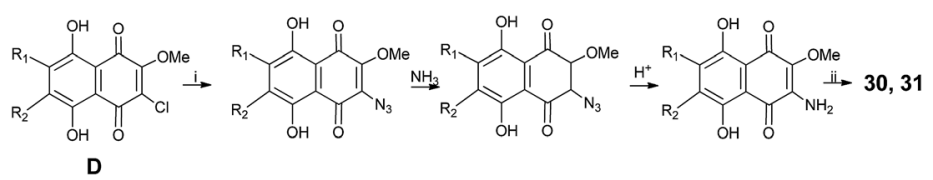

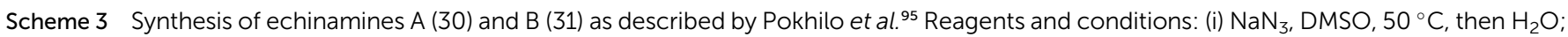
(ii) $\mathrm{HBr}-\mathrm{HOAC}$, reflux. 
<smiles>COc1c(O)c2c(c(O)c1OC)C(=O)C(O)=CC2=O</smiles>

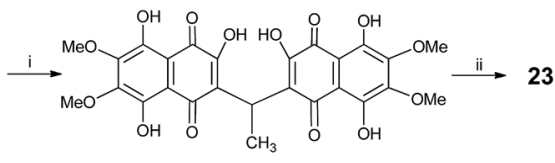

Scheme 4 Synthesis of bis(trihydroxynaphthazarin) (23). ${ }^{98}$ Reagents and conditions: (i) $\mathrm{MeNH}_{2} \cdot \mathrm{HCl}$, EtOH, heating $4 \mathrm{~h}$; (ii) $\mathrm{AlCl}_{3}, \mathrm{PhNO}_{2}$, $70{ }^{\circ} \mathrm{C}, 12 \mathrm{~h}$.

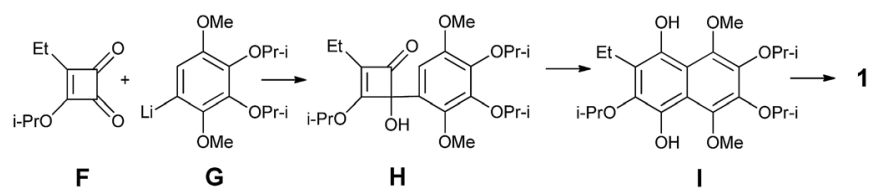

Scheme 5 Synthesis of echinochrome A (1) as described by PeñaCabrera and Liebeskind. ${ }^{99}$

(Scheme 2). Shestak et al. ${ }^{92}$ have reported synthesis of spinochromes $\mathrm{C}$ and $\mathrm{D}$ in gram quantities.

Echinamines A and B have been synthesized from chloromethoxynaphthazarin derivatives (D) by nucleophilic substitution of the chlorine atom with an azide group, followed by conversion to an amine and cleavage of the methoxy group by hydrogen bromide in acetic acid (Scheme 3). ${ }^{95}$

Pelageev et al. ${ }^{98}$ suggested a method for the preparative synthesis of bis(trihydroxynaphthazarins) using spinochrome $\mathrm{D}$ dimethyl ether (E). According to Scheme 4, compound $\mathbf{E}$ reacts with acetaldehyde under acidic conditions giving the tetramethoxy ether of bis(trihydroxynaphthazarin) (23). Demethylation of this product leads to bis(trihydroxynaphthazarin) (23) in good yield.

A simple and efficient total synthesis of echinochrome A starting with esters of squaric acid was developed by PeñaCabrera and Liebeskind ${ }^{99}$ according to Scheme 5. Key intermediates $\mathbf{F}$ and $\mathbf{G}$ were efficiently prepared from diisopropyl squarate, a common and readily available starting material. Nucleophilic addition of aryl-lithium $\mathbf{G}$ to $\mathbf{F}$, followed by thermal ring-expansion/cyclization of the 1,2-adduct $\mathbf{H}$, generated the hydroquinone $\mathbf{I}$. Oxidation and full deprotection of $\mathbf{I}$ gave the target compound with a yield of $41 \%$.

\section{Conclusion and outlook}

Although the chemistry of spinochrome pigments has been studied for more than 100 years, many questions still remain. For example, the fact that the functional value of spinochromes to sea urchins and their embryos is still unknown, highlights the need for further biochemical studies. This review aimed to summarize all existing data on spinochrome biosynthesis and provide an indication as to where there are still controversial opinions on this subject.

The challenges associated with the identification and structure elucidation of spinochromes have nowadays been largely overcome by using high-resolution mass spectrometry and 2DNMR methods. However, there are still many compounds of the spinochrome class for which NMR signal assignment has yet to be reported. Additionally, there is a need to develop more efficient methods for production of purified spinochromes, either from the natural sources or by chemical synthesis. Traditional extraction of spinochromes from natural sources involving organic solvents potentially exposes personnel and the environment to toxic compounds. The use of macroporous resin based extraction systems has the potential to reduce the amount of organic solvents used, however further investigation of the mechanism of action of these absorbents is required in relation to the extraction yield of spinochromes obtained.

This review indicates that the structure-function relationship of spinochromes still requires more investigation. Despite the growing number of investigations, a clear understanding of the relationship between the structure of spinochromes and their bioactivities is yet to be fully elucidated. To date many of the bioactivities reported for spinochromes have been determined using crude extracts. There is a need for determination of the biological activities of individual spinochromes and their analogues. This will enable exploration of opportunities to maximize commercial and scientific use of these compounds, as illustrated with the development of the 'Histochrome' product.

\section{Conflicts of interest}

There are no conflicts to declare.

\section{Acknowledgements}

The first author acknowledges the University of Otago for awarding a PhD scholarship to Yakun Hou.

\section{Notes and references}

1 X. Zhang and K. S. Vecchio, Front. Mater. Sci., 2013, 7, 103117.

2 A. Shavandi, V. Wilton and A. E.-D. A. Bekhit, J. Taiwan Inst. Chem. Eng., 2016, 65, 437-443.

3 O. N. Pozharitskaya, A. N. Shikov, M. N. Makarova, S. A. Ivanova, V. M. Kosman, V. G. Makarov, V. Bazgier, K. Berka, M. Otyepka and J. Ulrichová, Planta Med., 2013, 79, 1698-1704.

4 A. N. Shikov, V. I. Ossipov, O. Martiskainen, O. N. Pozharitskaya, S. A. Ivanova and V. G. Makarov, J. Chromatogr. A, 2011, 1218, 9111-9114.

5 D. Zhou, L. Qin, B.-W. Zhu, X.-D. Wang, H. Tan, J.-F. Yang, D.-M. Li, X.-P. Dong, H.-T. Wu and L.-M. Sun, Food Chem., 2011, 129, 1591-1597.

6 S. Soleimani, M. Yousefzadi, S. Moein, H. Rezadoost and N. A. Bioki, Med. Chem. Res., 2016, 25, 1476-1483.

7 H. Anderson, J. Mathieson and R. Thomson, Comp. Biochem. Physiol., 1969, 28, 333-345.

8 A. N. Shikov, O. N. Pozharitskaya, A. S. Krishtopina and V. G. Makarov, Phytochem. Rev., 2018, 1-26.

9 C. MacMunn, Q. J. Microsc. Sci., 1885, 25, 469-490. 
10 R. Kuhn and K. Wallenfels, Eur. J. Inorg. Chem., 1940, 73, 458-464.

11 R. E. Moore, H. Singh and P. J. Scheuer, J. Org. Chem., 1966, 31, 3645-3650.

12 R. E. Moore, H. Singh and P. J. Scheuer, Tetrahedron Lett., 1968, 9, 4581-4583.

13 J. Mathieson and R. Thomson, J. Chem. Soc. C, 1971, 153160.

14 T. Mukai, Bull. Chem. Soc. Jpn., 1960, 33, 453-456.

15 A. Y. Yakubovskaya, N. Pokhilo, N. Mishchenko and V. Anufriev, Russ. Chem. Bull., 2007, 56, 819-822.

16 N. P. Mischenko, S. A. Fedoreyev, N. D. Pokhilo, V. P. Anufriev, V. A. Denisenko and V. P. Glazunov, J. Nat. Prod., 2005, 68, 1390-1393.

17 E. A. Vasileva, N. P. Mishchenko, P. A. Zadorozhny and S. A. Fedoreyev, Nat. Prod. Commun., 2016, 11, 821-824.

18 R. Thomson, Naturally occurring quinones, Academic press, London and New York, 2nd edn, 1971.

19 T. Goodwin and S. Srisukh, Biochem. J., 1950, 47, 69-76.

20 N. Shinsaku, Y. Inouye and H. Nishikawa, Chem. Pharm. Bull., 1967, 15, 380-390.

21 E. A. Kol'tsova, G. N. Chumak and O. B. Maksimov, Chem. Nat. Compd., 1977, 13, 174-177.

22 E. Kol'tsova and O. Maximov, Chem. Nat. Compd., 1981, 1, 115.

23 E. A. Vasileva, N. P. Mishchenko and S. A. Fedoreyev, Chem. Biodiversity, 2017, 14, e1700182.

24 N. Utkina, A. Shchedrin and O. Maksimov, Chem. Nat. Compd., 1976, 12, 387-389.

25 E. Kol'tsova, V. Denisenko and O. Maksimov, Chem. Nat. Compd., 1978, 14, 371-374.

26 E. Vasileva, N. Mishchenko, H. M. N. Vo, L. M. Bui, V. Denisenko and S. Fedoreyev, Chem. Nat. Compd., 2017, 53, 356-358.

27 N. P. Mishchenko, E. A. Vasileva and S. A. Fedoreyev, Tetrahedron Lett., 2014, 55, 5967-5969.

28 C. Powell, A. Hughes, M. Kelly, S. Conner and G. McDougall, LWT-Food Sci. Technol., 2014, 59, 455-460.

29 A. Salaque, M. Barbier and E. Lederer, Bull. Soc. Chim. Biol., 1967, 49, 841-848.

30 C. Calestani, J. P. Rast and E. H. Davidson, Development, 2003, 130, 4587-4596.

31 N. V. Ageenko, K. V. Kiselev and N. A. Odintsova, J. EvidenceBased Complementary Altern. Med., 2011, 2011, 730356, 9 pages.

32 K. M. Herrmann and L. M. Weaver, Annu. Rev. Plant Biol., 1999, 50, 473-503.

33 A. L. Demain and A. Fang, Hist. Mod. Biotech. I, 2000, vol. 69, pp. 1-39.

34 S. Stekhova, E. Shentsova, E. Kol'tsova and N. Kulesh, Antibiot. Chemother., 1988, 33, 831-833.

35 L. Brasseur, E. Hennebert, L. Fievez, G. Caulier, F. Bureau, L. Tafforeau, P. Flammang, P. Gerbaux and I. Eeckhaut, Mar. Drugs, 2017, 15, E179.

36 T. Haug, A. K. Kjuul, O. B. Styrvold, E. Sandsdalen, Ø. M. Olsen and K. Stensvåg, J. Invertebr. Pathol., 2002, 81, 94-102.
37 M. Service and A. C. Wardlaw, Comp. Biochem. Physiol. B, 1984, 79, 161-165.

38 S. Soleimani, M. Yousefzadi and S. Moe, J. Kerman Univ. Med. Sci., 2015, 22, 614-628.

39 A. A. T. Sime, GB Pat., 2159056A, 1985.

40 A. Tyler, Proc. Natl. Acad. Sci. U.S.A, 1939, 25, 523-528.

41 D. C. White, J. Biol. Chem., 1965, 240, 1387-1394.

42 R. A. Boolootian, Am. Zool., 1962, 2, 275-284.

43 A. Drozdov and V. Vinnikova, Russ. J. Dev. Biol., 2010, 41, 3745.

44 T. Kominami, H. Takata and M. Takaichi, Dev., Growth Differ., 2001, 43, 699-707.

45 P. C. Branco, J. C. S. Borges, M. F. Santos, B. E. J. Junior and J. R. M. C. da Silva, Mar. Environ. Res., 2013, 92, 95-101.

46 P. C. Branco, L. N. Pressinotti, J. C. S. Borges, R. S. Iunes, J. R. Kfoury, M. O. da Silva, M. Gonzalez, M. F. dos Santos, L. S. Peck and E. L. Cooper, Polar Biol., 2012, 35, 221-229.

47 S.-S. Suh, J. Hwang, M. Park, S. Y. Park, T. K. Ryu, S. Lee and T.-K. Lee, Ecotoxicol. Environ. Saf., 2014, 109, 63-69.

48 A. Pinsino and V. Matranga, Dev. Comp. Immunol., 2015, 49, 198-205.

49 A. Kroh and R. Mooi, 2018, http:/www.marinespecies.org/ echinoidea.

50 R. Kuwahara, H. Hatate, T. Yuki, H. Murata, R. Tanaka and Y. Hama, LWT-Food Sci. Technol., 2009, 42, 1296-1300.

51 D. M. Li, D. Y. Zhou, B. W. Zhu, L. Miao, L. Qin, X. P. Dong, X. D. Wang and Y. Murata, Eur. Food Res. Technol., 2013, 237, 331-339.

52 E. Kol' tsova and N. Krasovskaya, Chem. Nat. Compd., 2009, 45, 427-428.

53 A. Bazhin, Russ. J. Mar. Biol., 2002, 28, 298-307.

54 D. Tatarenko and A. Poltaraus, Zool. Zh., 1993, 72, 61-72.

55 Y. Agatsuma, Developments in Aquaculture and Fisheries, Science, 2001, 32, 347-362.

56 X. Zhou, K. Wen, X. Yang, R. Huang, G. Dong, B. Yang, J. Sun and Y. Liu, Biochem. Syst. Ecol., 2010, 38, 103-105.

57 M. S. Kelly, A. D. Hughes and E. J. Cook, Developments in Aquaculture and Fisheries, Science, 2007, 37, 287-295.

58 Y. Agatsuma, Developments in Aquaculture and Fisheries, Science, 2007, 37, 459-472.

59 S. S. Dautov and S. Kashenko, Russ. J. Mar. Biol., 2008, 34, 415-420.

60 D. E. Walker and J.-M. Gagnon, J. Mar. Biol., 2014, 2014, 297631, 9 pages.

61 R. Kuwahara, H. Hatate, A. Chikami, H. Murata and Y. Kijidani, LWT-Food Sci. Technol., 2010, 43, 1185-1190.

62 R. Amarowicz, J. Synowiecki and F. Shahidi, Food Chem., 1994, 51, 227-229.

63 K. Nishibori, Nature, 1959, 184, 1234.

64 D. Becher, C. Djerassi, R. E. Moore, H. Singh and P. J. Scheuer, J. Org. Chem., 1966, 31, 3650-3660.

65 I. Singh, R. Moore, C. Chang, R. Ogata and P. Scheuer, Tetrahedron, 1968, 24, 2969-2978.

66 R. E. Moore, H. Singh, C. W. J. Chang and P. J. Scheuer, Tetrahedron, 1967, 23, 3271-3305.

67 V. Glazunov and D. Berdyshev, J. Appl. Spectrosc., 2014, 81, 553-564. 
68 A. Gerasimenko, S. Fedoreyev and N. Mischenko, Crystallogr. Rep., 2006, 51, 42-46.

69 J. Bowie, D. Cameron and D. Williams, J. Am. Chem. Soc., 1965, 87, 5094-5099.

70 D. Y. Zhou, B. W. Zhu, X. D. Wang, L. Qin, D. M. Li, L. Miao and Y. Murata, Int. J. Food Sci. Technol., 2012, 47, 1479-1486.

71 A. V. Lebedev, M. V. Ivanova and E. K. Ruuge, Arch. Biochem. Biophys., 2003, 413, 191-198.

72 A. N. Shikov, V. I. Ossipov, M. Karonen, O. N. Pozharitskaya, A. S. Krishtopina and V. G. Makarov, Nat. Prod. Res., 2017, 31, 1747-1751.

73 N. P. Mishchenko, S. A. Fedoreev and V. L. Bagirova, Pharm. Chem. J., 2003, 37, 48-52.

74 D. Berdyshev, V. Glazunov and V. Novikov, Russ. Chem. Bull., 2007, 56, 413-429.

75 A. Lebedev, E. Levitskaya, E. Tikhonova and M. Ivanova, Biochemistry (Moscow), 2001, 66, 885-893.

76 E. A. Vasileva and N. P. Mishchenko, International Conference and Exhibition on Marine Drugs and Natural Products, July 2527, 2016, Melbourne, Australia, vol. 1000, 483.

77 K. Mizutani, T. Nagatsu, M. Asashima and S. Kinoshita, Biochem. Pharmacol., 1972, 21, 2463-2468.

78 H. Kuzuya, K. Ikuta and T. Nagatsu, Biochem. Pharmacol., 1973, 22, 2772-2774.

79 S. A. Ivanova, O. N. Pozharitskaja, A. N. Shikov, M. N. Makarova and V. G. Makarov, RU Pat., 2488402C1, 2013.

80 I. G. Agafonova, V. N. Kotelnikov, N. P. Mischenko and N. G. Kolosova, Bull. Exp. Biol. Med., 2011, 150, 739-743.

81 E. Egorov, V. Alekhina, T. Volobueva, S. Fedoreev, N. Mishchenko and E. Kol'tsova, Vestn. Oftalmol., 1998, 115, 34-35.

82 E. N. Kareva, D. A. Tikhonov, N. P. Mishchenko, S. A. Fedoreev and N. L. Shimanovskii, Pharm. Chem. J., 2014, 48, 149-152.

83 I. Agafonova, R. Bogdanovich and N. Kolosova, Bull. Exp. Biol. Med., 2015, 160, 223-227.

84 O. Lebed'ko, B. Y. Ryzhavskii and O. Demidova, Bull. Exp. Biol. Med., 2015, 159, 351-354.
85 S. H. Jeong, H. K. Kim, I. S. Song, S. J. Lee, K. S. Ko, B. D. Rhee, N. Kim, N. P. Mishchenko, S. A. Fedoryev, V. A. Stonik and J. Han, Mar. Drugs, 2014, 12, 2922-2936.

86 D. Y. Seo, R. A. McGregor, S. J. Noh, S. J. Choi, N. P. Mishchenko, S. A. Fedoreyev, V. A. Stonik and J. Han, Mar. Drugs, 2015, 13, 5722-5731.

87 H. K. Kim, J. B. Youm, S. H. Jeong, S. R. Lee, I.-S. Song, T. H. Ko, J. R. Pronto, K. S. Ko, B. D. Rhee and N. Kim, Pflugers Arch., 2015, 467, 2151-2163.

88 S. R. Lee, J. R. D. Pronto, B.-E. Sarankhuu, K. S. Ko, B. D. Rhee, N. Kim, N. P. Mishchenko, S. A. Fedoreyev, V. A. Stonik and J. Han, Mar. Drugs, 2014, 12, 3560-3573.

89 A. S. Mohamed, A. M. Soliman and M. A. S. Marie, Life Sciences, 2016, 151, 41-49.

90 I. Singh, R. E. Moore, C. W. Chang and P. J. Scheuer, J. Am. Chem. Soc., 1965, 87, 4023-4024.

91 V. P. Anufriev, V. L. Novikov, O. B. Maximov, G. B. Elyakov, D. O. Levitsky, A. V. Lebedev, S. M. Sadretdinov, A. V. Shvilkin, N. I. Afonskaya and M. Y. Ruda, Bioorg. Med. Chem. Lett., 1998, 8, 587-592.

92 O. P. Shestak, V. P. Anufriev and V. L. Novikov, Nat. Prod. Commun., 2014, 9, 953-956.

93 N. Pokhilo and V. Anufriev, Chem. Nat. Compd., 2016, 52, 939-940.

94 K. Borisova and V. Anufriev, Chem. Nat. Compd., 2012, 48, 202-204.

95 N. D. Pokhilo, M. I. Shuvalova, M. V. Lebedko, G. I. Sopelnyak, A. Y. Yakubovskaya, N. P. Mischenko, S. A. Fedoreyev and V. P. Anufriev, J. Nat. Prod., 2006, 69, 1125-1129.

96 G. I. Melman, K. L. Borisova, N. D. Pokhilo, V. V. Makhankov and V. P. Anufriev, Tetrahedron Lett., 2016, 57, 736-738.

97 N. D. Pokhilo, G. I. Melman, M. I. Kiseleva, V. A. Denisenko and V. P. Anufriev, Nat. Prod. Commun., 2015, 10, 1243-1246.

98 D. N. Pelageev and V. P. Anufriev, Synthesis, 2016, 48, 761764.

99 E. Peña-Cabrera and L. S. Liebeskind, J. Org. Chem., 2002, 67, 1689-1691. 